\title{
Three Dimensional NURBS Modeling and Fabrication of Rotorcraft Design
}

\author{
Geoffrey A. Ament ${ }^{1}$ \\ NASA Ames Research Center, Mountain View CA, 94035 \\ University of Wisconsin Platteville, Platteville WI, 54115
}

\begin{abstract}
Before an aircraft is put into production, it must undergo a variety of tests inside a wind tunnel. Rather than performing these tests on a full-scale plane, scaled models are often utilized. Scaled models allow an aircraft to be tested in smaller facilities, can be built quickly, and can save money, as long as the physics being studied scale correctly. Another use for aircraft models is to demonstrate how a model aircraft is built and tested in a wind tunnel, showing aircraft interior and how the exterior mechanics operate. The objective of this project was to show the steps involved in building a model aircraft and what these models can be used for. The model aircrafts worked on and mentioned in this paper include the Large Civil Tilt Rotor (LCTR2) and XV-15 Tilt Rotor Research Aircraft. Models are either computer modeled from blueprints or provided by our mentor, Eduardo Solis. The models are constructed using the program Rhino, prototyped in a MakerBot 3D printer, and finally 3D printed using the Dimension Elite printer and a laser cutter. The models do not have real working engines; they are only for display and educational purposes; the models only show only how the model airframes are built using the stated 3D printers and laser cutters. Four engineering interns worked on this project. As Project Leader, I distributed the workload for the three major projects - the LCTR2 model, the XV-15 RotCFD model, and the 1/6scale model of the 7-Foot by 10-Foot Wind Tunnel.
\end{abstract}

\section{Nomenclature}

$\begin{array}{ll}\text { NURBS } & =\text { Non-Uniform Rational Basis Spline } \\ \text { LCTR(2) } & \text { Large Civil Tilt Rotor (2) } \\ \text { VTOL } & =\text { Vertical Take Off and Landing } \\ \text { XV-15 } & =\text { XV-15 Tilt Rotor Aircraft } \\ \text { Rhino } & =\text { Rhinoceros 5 3D NURBS Modeling Software } \\ \text { RotCFD } & =\text { Computational Fluid Dynamics Software }\end{array}$

\section{Introduction}

To help adapt to a crowded airspace, the LCTR2 and XV-15 were designed to allow for vertical takeoff. This design allows for efficient use of aircraft runways, allowing more aircraft to land in airports. Before these aircraft designs can be put into production, they must undergo a series of tests. Instead of running these tests on a full scale aircrafts, models are created to quickly and inexpensively test an aircraft in a wind or water tunnel. Another use of aircraft models is to demonstrate how the tested or full scale models operate. This paper will illustrate the design procedure that goes into building model LCTR2 and XV-15 rotorcrafts for demonstration and testing purposes.

\footnotetext{
${ }^{1}$ Thanks to Wisconsin Space Grant Consortium for making this research opportunity possible. Special thanks to my mentors William Warmbrodt and Eduardo Solis for their support throughout the project.
} 


\section{Project Background}

LCTR2 Background. The NASA Heavy Lift Rotorcraft Systems Investigation evaluated various aircraft designs to determine which rotorcraft system could be flown safely for the civil mission. With that said, because of the expected $100,000 \mathrm{lb}$ aircraft weight traveling at 350 knots, designing such an aircraft could prove difficult. After investigation, the Large Civil Tilt Rotor (LCTR) proved to be the most promising aircraft for performance, stability and safety (1). The goal of these aircraft is to compete with current jet aircraft and help a crowded airspace. The aircraft is to carry 120 passengers with baggage, travel $1200 \mathrm{~nm}$, and travel mach 0.6 at 30,000 feet (3). A picture of the LCTR aircraft can be seen in Figure 1.

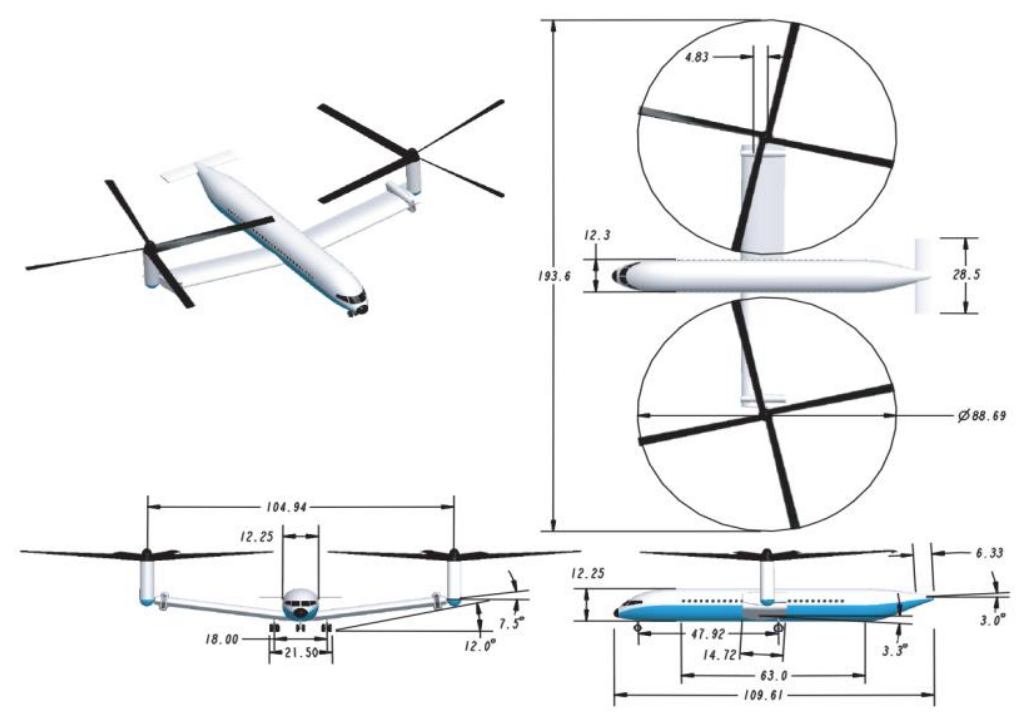

Figure 1. Shows the LCTR concept model. All dimensions shown are in $\mathrm{ft}$ and degrees (2).

In April 2012, the LCTR was first tested in the NASA Ames 7-Foot by 10-Foot Wind Tunnel. The model was tested in airplane mode as well as helicopter mode; in helicopter mode, the nacelles were rotated between 60 and 95 degrees and tested up to 80 knots. A much smaller model was tested in both modes in the NASA Ames Fluid Mechanics Laboratory Water Tunnel. Picture of the models tested in air and in water in these tunnels can be seen in Figures 2.
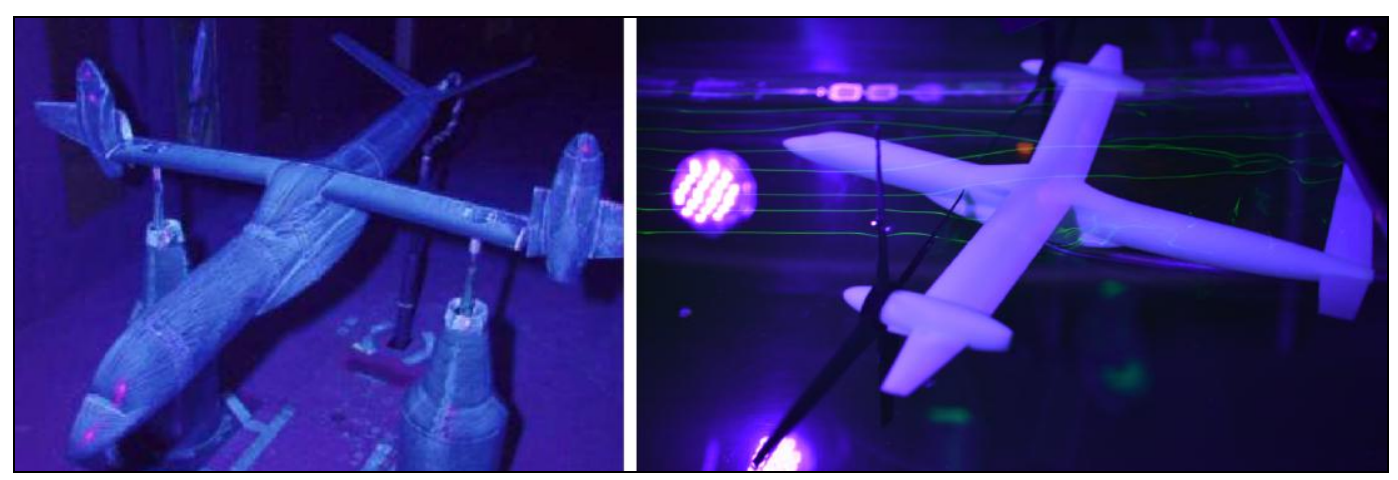

Figure 2. Left, LCTR2 model tested in 7x10 ft wind tunnel. Right, LCTR2 model tested in water tunnel. 
XV-15 History. The XV-15 vertical takeoff and landing aircraft would be ideal for short haul, civilian transport air travel. A picture of this aircraft can be seen in Figure 3 (1). In 1997, an fullscale aero acoustic test was performed on the XV-15 rotor at the NASA Ames Research Center to analyze tilt rotor noise with the goal of reducing it; the test was conducted in the 80- by 120Foot Wind Tunnel test section. To help reduce noise, the number of rotors blades were increased from three to four.

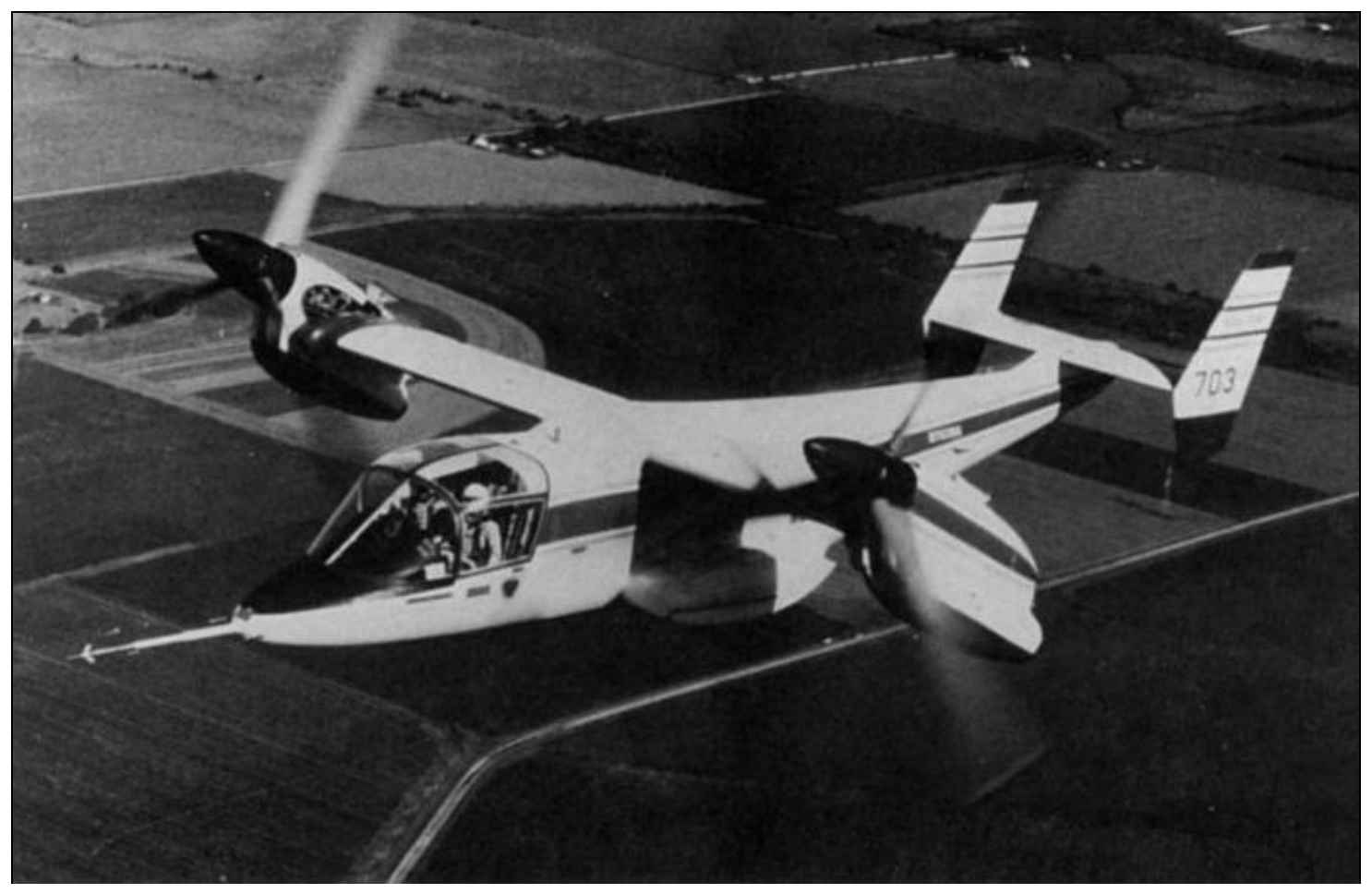

Figure 3. Shows a picture of the XV-15.

\section{Design Process}

Rhino Program and Machine Tools. Before starting physical construction of the model aircraft, the models had to be digitalized using the 3D modeling program Rhino. Rhino has the ability to render models to provide a visual of how the physical model will look. Once a digital version of the aircrafts was input into Rhino, the parts had to be $3 \mathrm{D}$ printed and / or laser cut. In preparation for fabrication, we were trained on these machines and other Space Shop equipment. With the combined knowledge of Rhino and other Space Shop equipment, the models were ready for $3 \mathrm{D}$ printing and assembly.

LCTR2 Model Design. The LCTR2 model built this summer was a scaled replica of the model tested in the 7 by $10 \mathrm{ft}$ wind tunnel. After a 1 to 1 scale model was digitalized in Rhino, the model was scaled down to be 1/44 of the original blueprint. Further, the model needed to showcase how the wind tunnel test model was built, demonstrating both interior and exterior construction. To accomplish this, the model needed to be 3D printed in sections; this allows the user to pull pieces off the LCTR2 model to view how the wind tunnel test model was built internally and externally. For demonstration purposes, only half of the aircraft can be pulled 
apart; the second half of the aircraft is completely intact. A overview of the LCTR2 model, assembled and pulled apart can be seen in Figure 4.

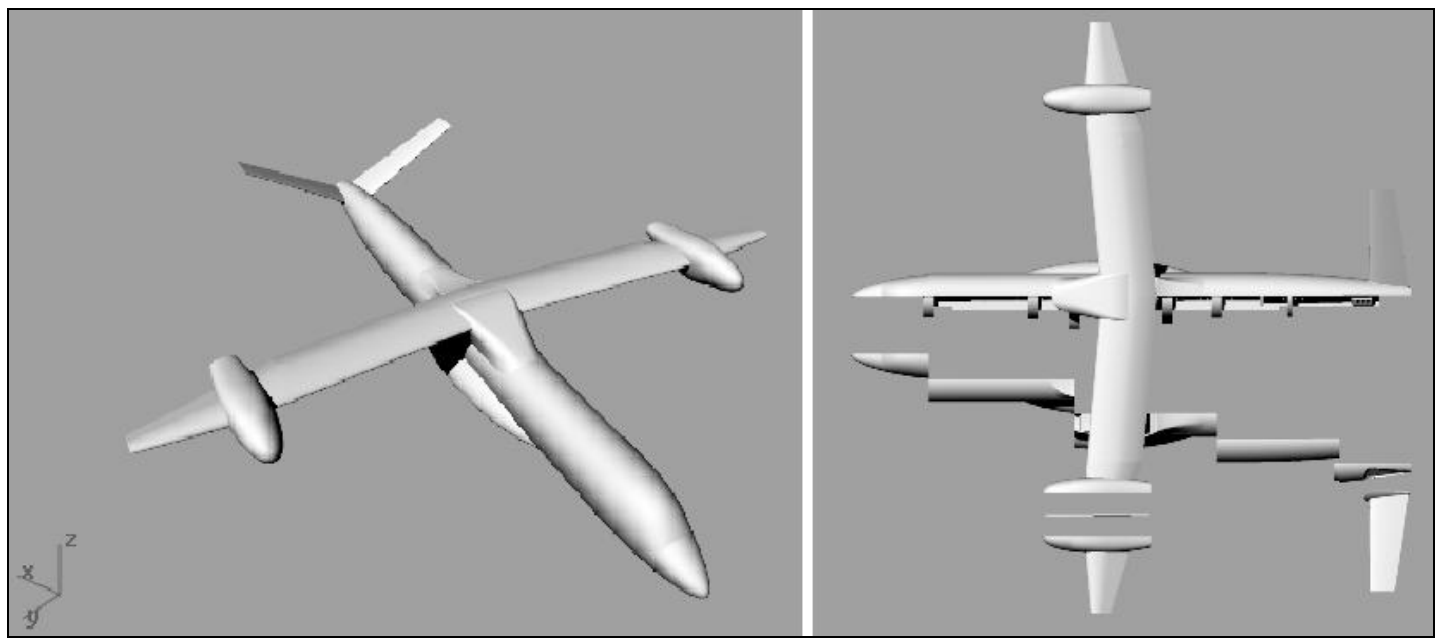

Figure 4. Left, shows a rendered view of the 1/44 scale LCTR2 in Rhino. Right, shows how the rendered model can be pulled apart.

Before the model could be printed, the pull apart pieces needed to be designed such that they could easily be detached from the model (Figure 4). To accomplish this, pin inserts were implemented on each detachable piece; a 3D model showing these pin hole attachments can be seen on the central body piece of the LCTR2 model in Figure 5.

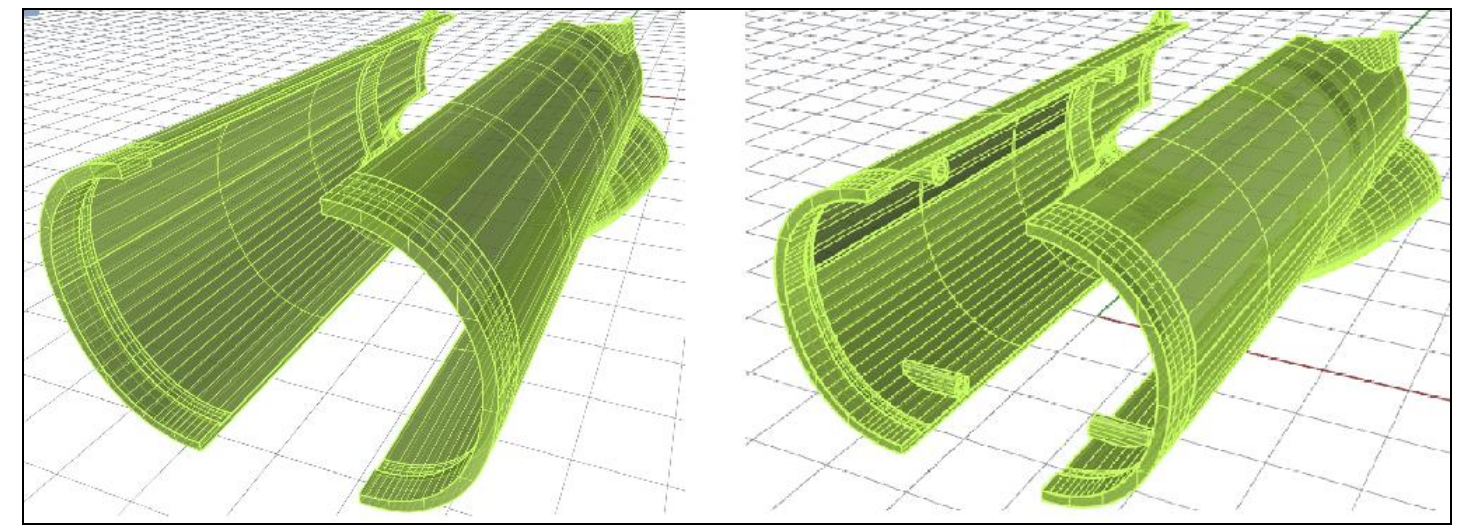

Figure 5. On left, notice the original model had no way of fastening one side of the model body to the other. On right, the left design was upgraded to include pin hole inserts to attach or remove the model side panels.

Overall, the aircraft contains 50 plastic 3D printed pieces modeled in Rhino, 10 of which are detachable. The aircraft is held together by a 1.5 inch by 1.5 inch aluminum core. Once all 50 pieces were separated in the Rhino software, they were 3D printed using a high resolution 3D printer, the Dimension Elite. With that said, before printing on the Dimension Elite, some parts were prototyped on a MakerBot 3D printer; Figure 6 shows the LCTR2 3D printed parts. 


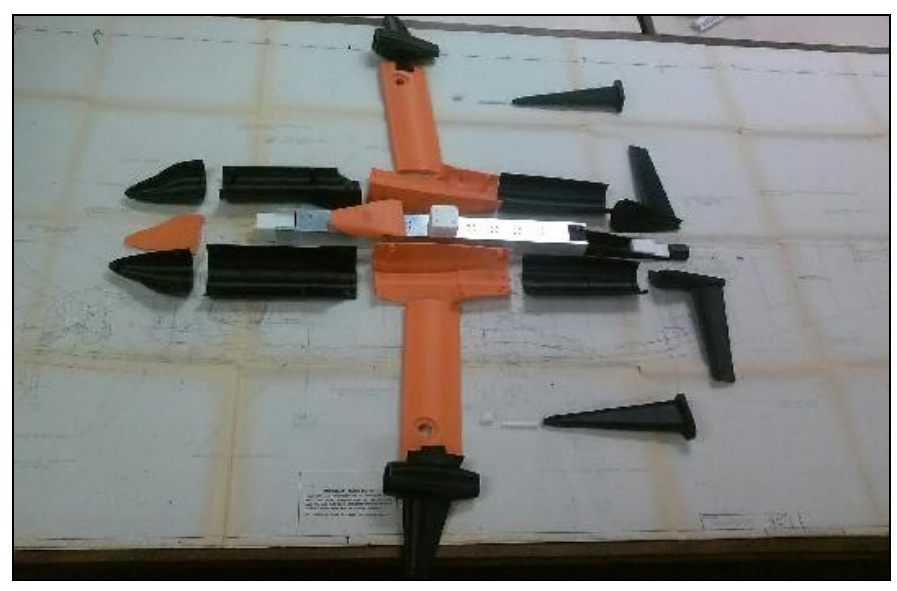

Figure 6. LCTR2 model 3D printed parts along with the aluminum core to hold parts together.

After each part was 3D printed, each piece was test fitted before fabrication. Once all pieces fit properly, each piece was sanded until smooth to touch; if a surface has imperfections, the piece required bondo and re-sanding of the area. Once all pieces were sanded, primer was applied to help fill any remaining imperfections in the model surfaces. Once dry, the pieces were then wet sanded. This process was repeated until the model was free of imperfections. Lastly, a black coat was added to give the LCTR2 model a matte finish. This fabrication process can be seen in Figures 7 and 8 .
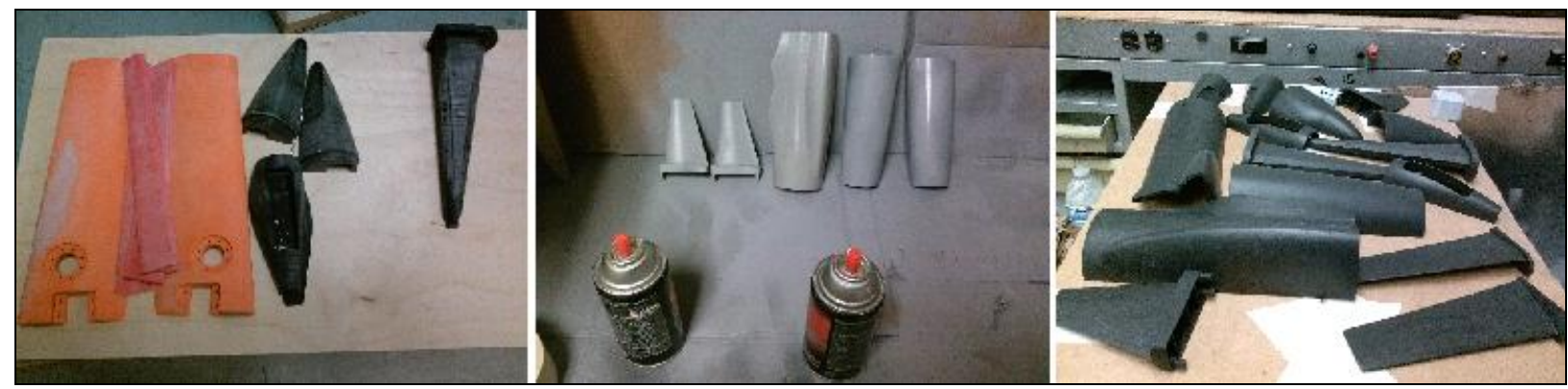

Figure 7. Left to right, shows initial sanding, application of primer and black paint to the 3D printed pieces.

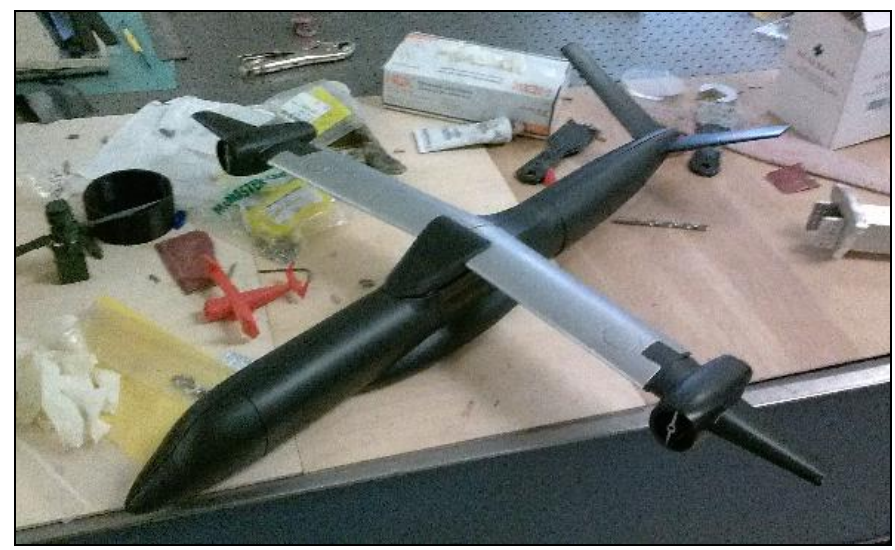

Figure 8. Shows an assembled model of the LCTR2 without attached blades. 
LCTR2 Rotational Base Design and LCTR2 Base Attachment. After the LCTR2 model was complete, the model was attached to a rotational base which allowed for 360 degree rotation as well as pitch angle of \pm 15 degrees via shepherds crook. To do this, the model needed to sit on two elevated columns which inserted into the rotational base. In addition, the shepherds crook needed to insert into the base, allowing for the shepherds crook rod to slide in and out of the base. A rendered view of the LCTR2 model mounted on the rotational base can be seen in Figure 9.

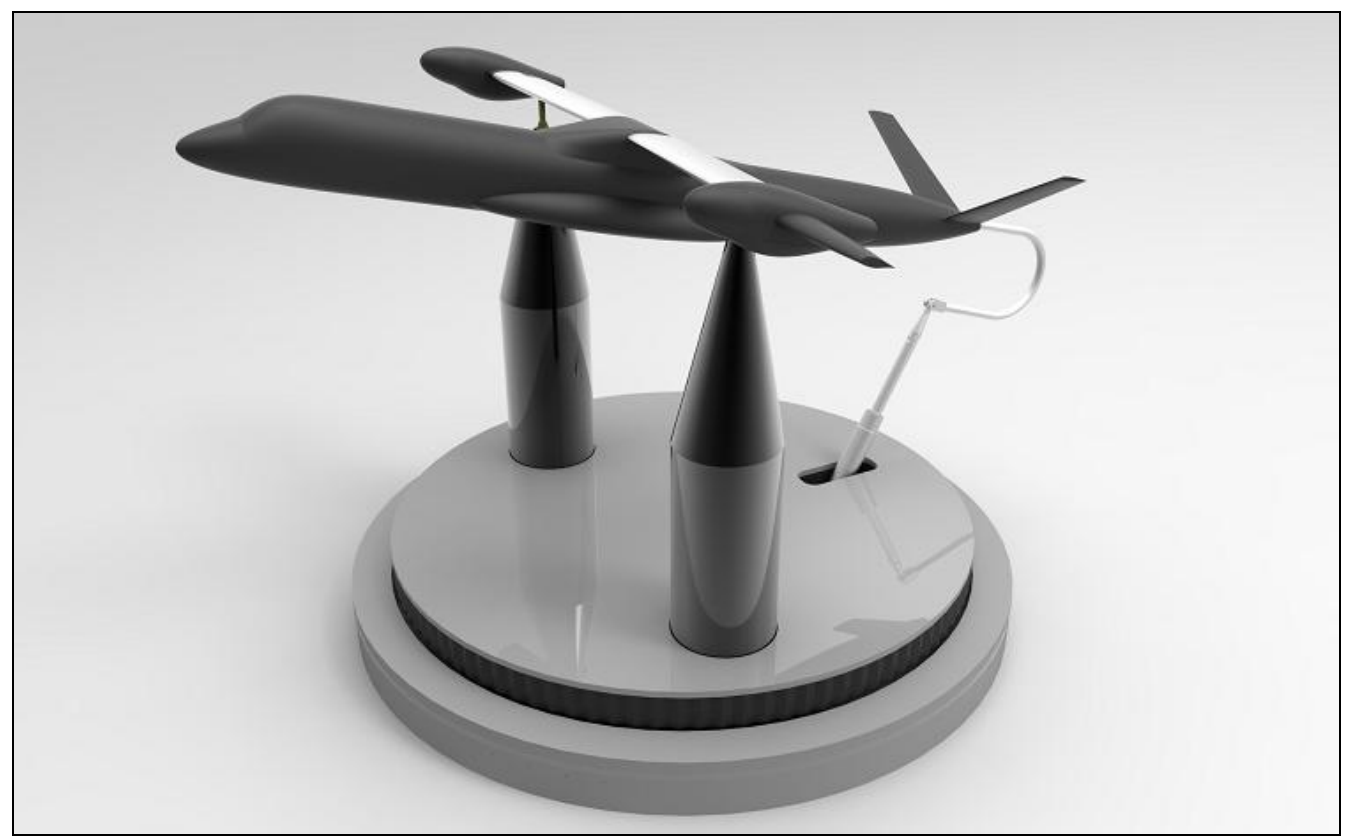

Figure 9. Rendered view of the $1 / 44$ scale LCTR2 model on a 360 degree rotational base.

The base was created using layers of acrylic with a few ABS plastic parts. Base dimensions can be seen in Figure 10; base parts cut by laser or 3D printed parts can be seen Figure 11.

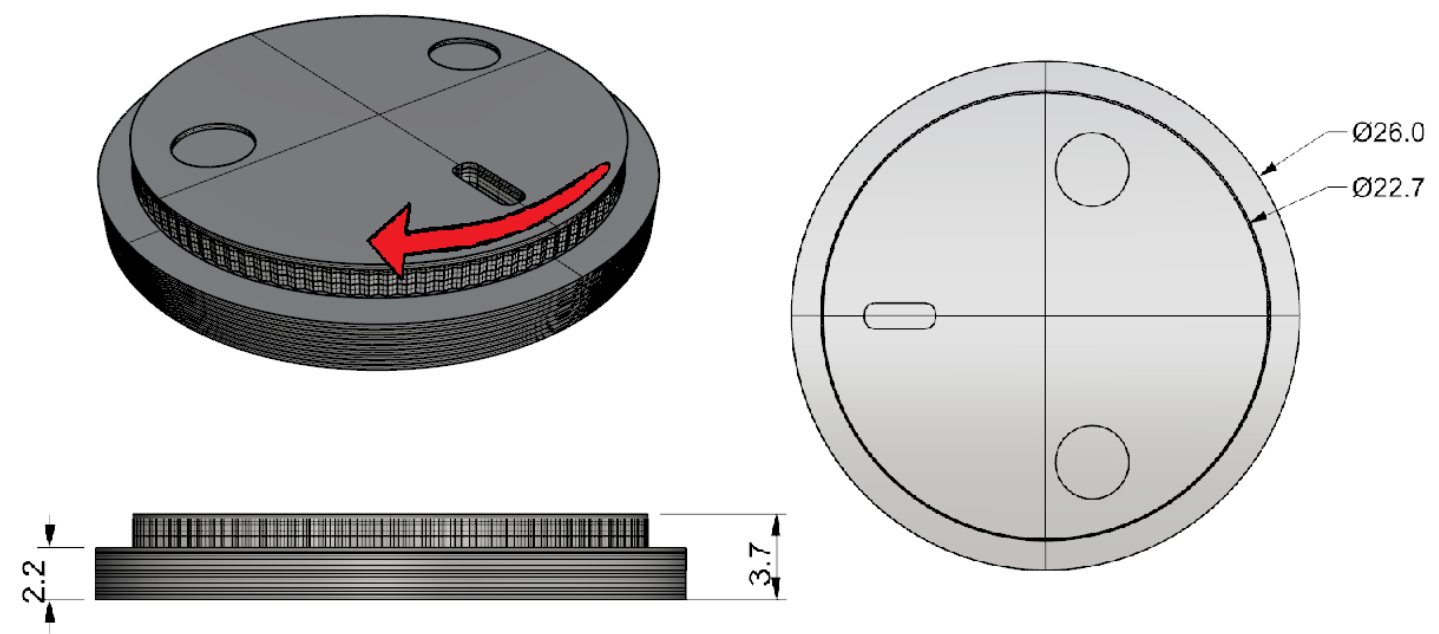

Figure 10. 360-degree LCTR rotational base, all dimensions shown are in inches. 

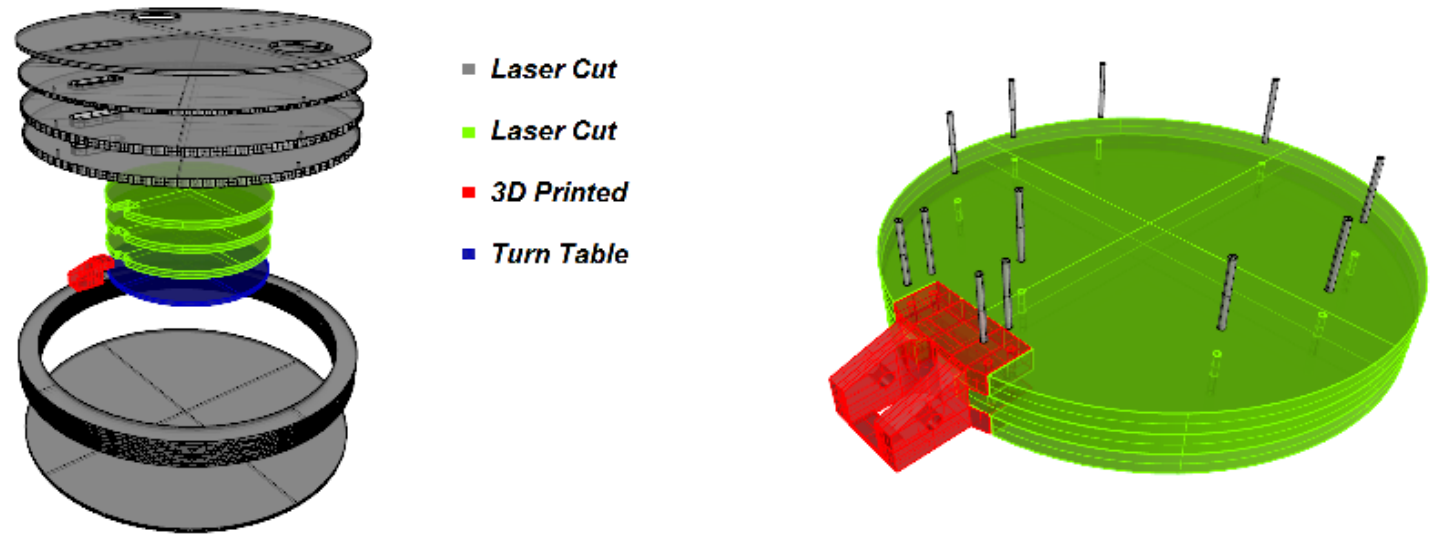

Figure 11. Left, shows exploded view of the laser cut and 3D printed parts. The blue round disk represents the turntable which rotates the model. Color coding is for illustration purposes only and does not represent the final product. Right, shows how the 3D printed parts and acrylic sheets where physically assembled together via $5 / 32$ inch pins.

Once the laser cut and 3D printed parts were ready, the parts were then sent through the same fabrication process as the LCTR2 model: sanding, wet sanding, application of bondo, and painting. Pictures of this process can be seen in Figures 12 through 14 .
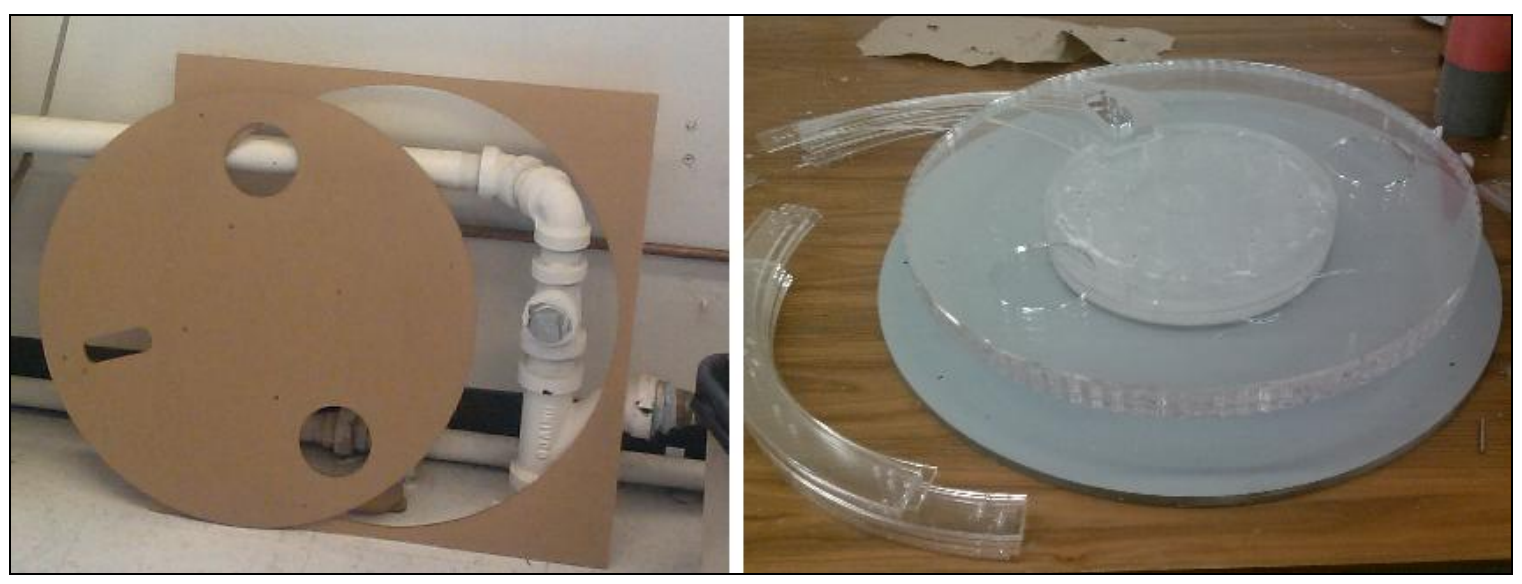

Figure 12. Left, shows the base being laser cut. Right, shows the assembly process of the laser cut pieces.

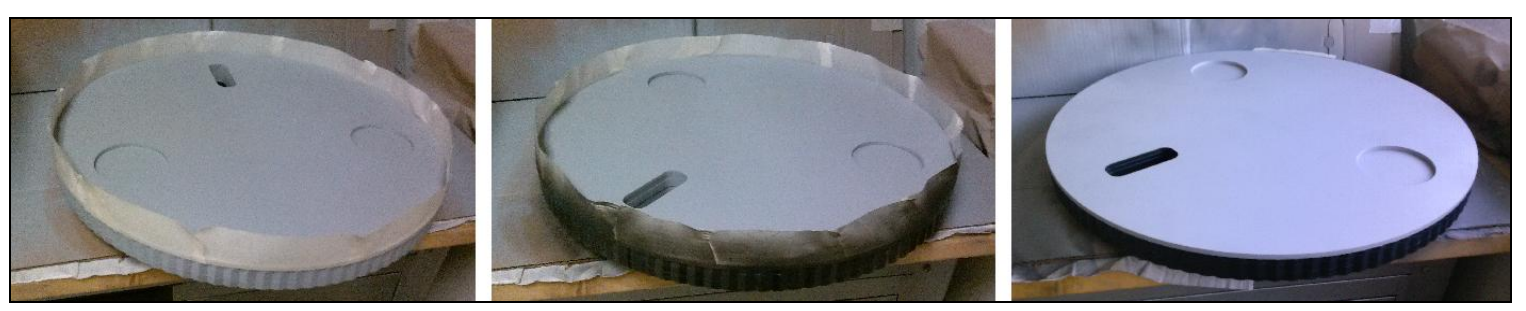

Figure 13. Shows the fabrication process: dry wet sanding, application of bondo, priming and painting the acrylic pieces. 


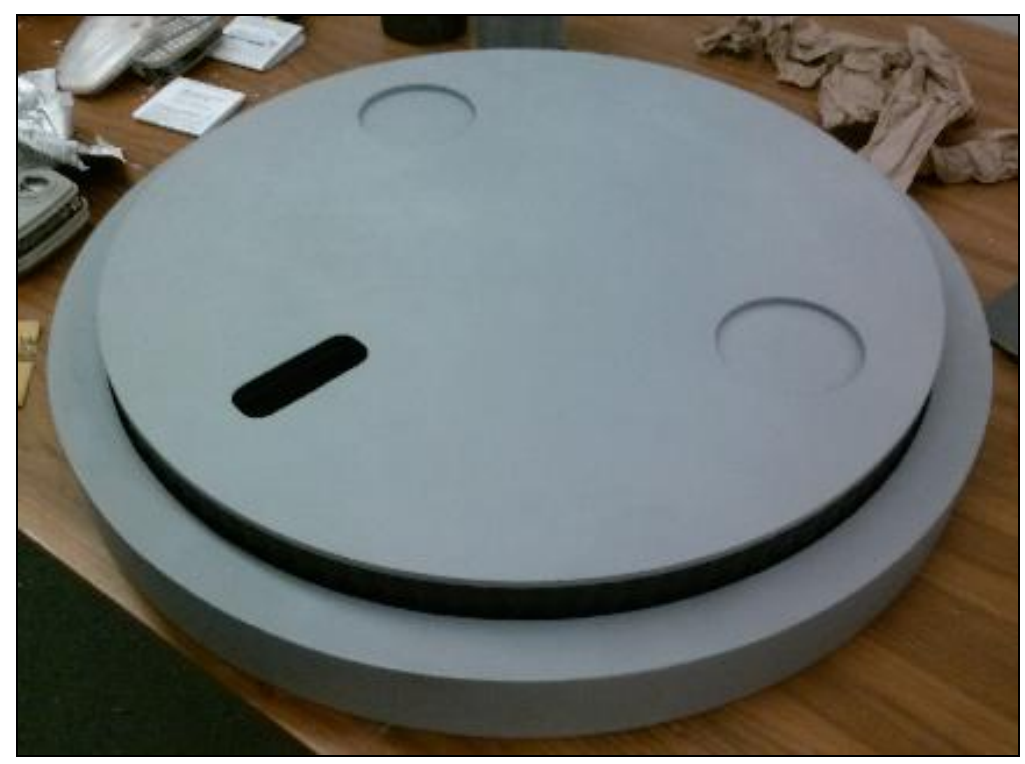

Figure 14. Shows the final assembly of fabricated base.

To allow the shepherds crook to pitch the model, two mechanical connection were implemented to connect the base with shepherds crook. The first connection (seen in red) uses a $1 / 4$ in diameter by 2 in pin to pivot the shepherds crook; it is mounted to the base (seen in green) which can be seen in Figure 15.

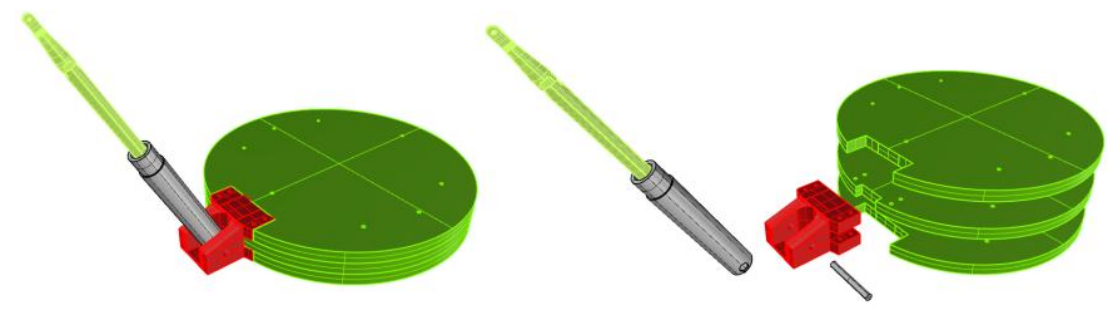

Figure 15. Mechanical connection (red) between shepherds crook (gray) and base (green).

The second mechanical connection allows the shepherds crook to easily change inclination angle. By pushing down or pulling up on the mechanism, the rod will be fixed / not fixed into position. This design allowed the user to intuitively and quickly change the model angle. Figure 16 shows the design process of 3D modeling, 3D printing and assembly.

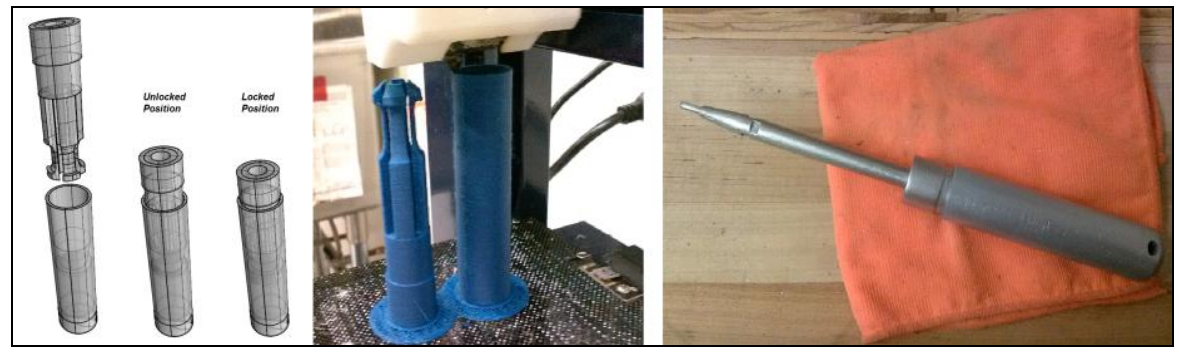

Figure 16. Left to right, shows the design process from 3D modeling, 3D printing to physical assembly. This mechanism allows the LCTR2 to be locked at a certain pitch angle. 
XV-15 Design Process for RotCFD Analysis. Similar to the LCTR2, the XV-15 was digitalized from blueprints into Rhino. With the LCTR2, the model had been previously digitalized by Eduardo Solis; what was left was to modify the existing files, print and fabricate the pieces. For this model, the XV-15 was started from scratch. However, the digitalized XV-15 would not be physically built, it would be used for RotCFD simulations; because the model was used for simulation rather than display, a XV-15 model could be quickly digitalized. To do this, images of the original blueprints needed to be inserted into the Rhino software; once the images were set, the blueprint images needed to be scaled to the actual size of the XV-15. After scaling is complete, A wireframe of the aircraft can be generated by tracing the curves according to the blueprint images. This general shape will allow a network surface to connect the wireframe edges to create the curved surfaces of the aircraft; this can be seen in Figure 17.

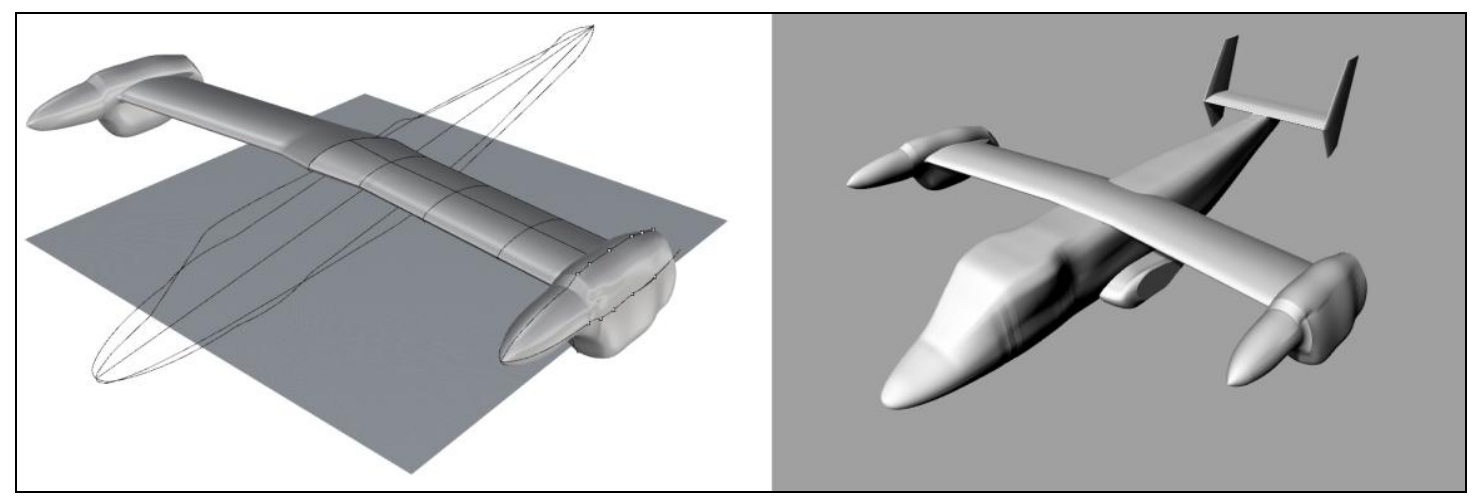

Figure17. Shows how the XV-15 aircraft was digitalized using the Rhino software.

Once the model was complete, the file was converted into an stl file to be analyzed in RotCFD. RotCFD is a program built to simulate rotorcraft / turbines and analyze performance. This program can run with low computational budget, that is, no need for a supercomputer. Figure 18 shows the $\mathrm{XV}-15$ running in the RotCFD simulation software.
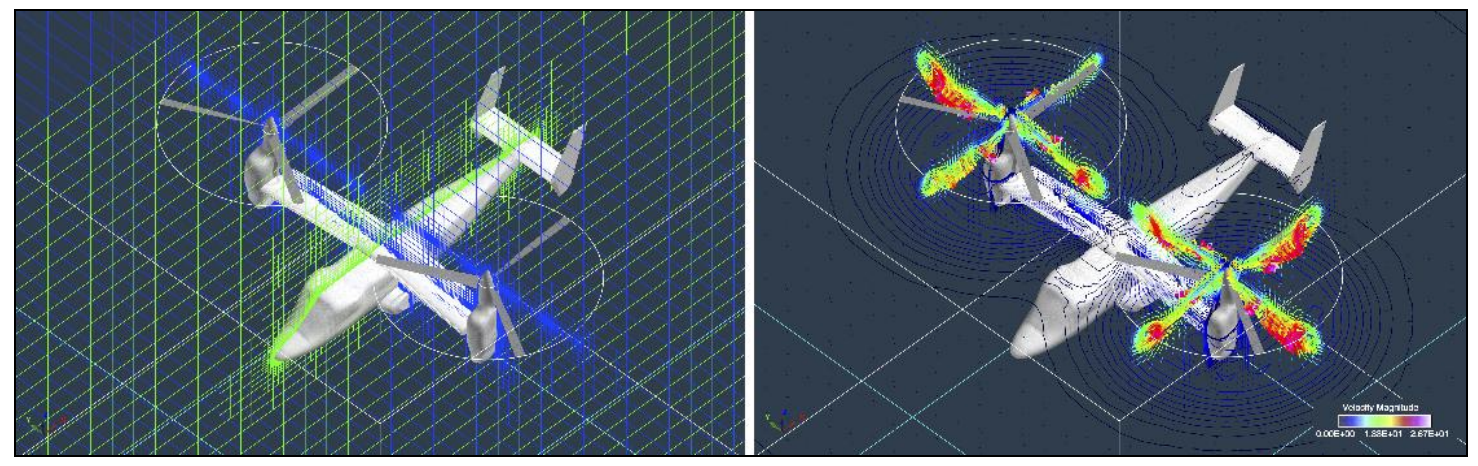

Figure 18. XV-15 model in RotCFD.

\section{Complications For all Projects}

The first complication was getting use to the software Rhino to build the models. Eduardo Solis was very helpful in providing tutorials and additional help when using Rhino. After a few weeks of work, I became familiar with Rhino and was ready for 3D printing the Rhino created parts. 
Because printing parts can be very time consuming, we had to manage our 3D print time and stay on it to print all parts before fabrication could take place. Thirdly, because there were multiple people working on the same model, we had to make sure everyone's computer model had the same scaled parts, or else our 3D printed pieces would not fit together. Lastly, because we were given multiple projects to complete, I organized a gantt chart so that group members could have a visual in how much time they have to finish their projects by end date.

\section{Conclusions}

A scale model of the LCTR2 aircraft (which rests on a rotational base), RotCFD model of the $\mathrm{XV}-15$, and a $1 / 6^{\text {th }}$ scale 7 -Foot by 10 -Foot Wind Tunnel model with a second LCTR2 model built to scale down to the $1 / 6^{\text {th }}$ scale wind tunnel were worked on throughout the internship program. These projects will provide the general public with a better understanding of how LCTR2 model was built for testing in the 7-Foot by 10- Foot Wind Tunnel, give a better understanding of how wind tunnels work and benefit aircraft development and improvement. The LCTR2 aircraft was completely modeled, fabricated and assembled showing how this model was build and used in the 7-Foot by 10 Foot Wind Tunnel test section. Second, a RotCFD model of the XV-15 was produced and is currently being used for aerodynamic analysis. Lastly, the $1 / 6^{\text {th }}$ scale wind tunnel has been designed and fabricated, but has yet to be assembled; long term, electronics will be incorporated into the Wind Tunnel model to show how the test section operates when running a test.

\section{Acknowledgments}

I would like to thank the Wisconsin Space Grant for funding this project, without them this would not have been possible. Special thanks to my mentors William Warmbrodt and Eduardo Solis for helping me along the way to complete my projects, thanks to Larry Young for helping me as I worked with the MakerBot. Special thanks to the workers at the Space Shop, Alex Mazhari, Randall Ticknor and Bryan Hackett for printing our parts throughout the summer. Lastly, thanks to Kristen Kallstrom for providing me the opportunity to work at NASA Ames Research Center for the summer.

\section{References}

${ }^{1}$ Ref. 1 - pdf, Solis, E., "General Information on 2105 Summer Internship Models", Mountain View, CA.

${ }^{2}$ Acree, C., "Impact of Technology on Heavy Lift Tiltrotors," Proceedings of the American Helicopter Society 62nd Annual Forum, Phoenix, AZ, May 9-11, 2006.

${ }^{3}$ Acree, W.C., "Integration of Rotor Aerodynamic Optimization with Conceptual Design of a Large Civil Tilt Rotor", Presented at the American Helicopter Society Specialists' Conference on Aeromechanics, January 20-22, 2010

${ }^{4}$ History.nasa.gov, 'Chapter 7 On the Eve of Shuttle (1973-1980),' Available: http://history.nasa.gov/SP-4406/chap7.html 